\title{
A Professional QoS Provisioning In The Intra Cluster Packet Level Resource Allowance For Wireless Mesh Networks
}

\author{
K.Senthamil Selvan ${ }^{1}$ and B. LakshmiDhevi ${ }^{2}$ \\ ${ }^{1}$ Department of Electronics and Communication Engineering, Research Scholar, \\ St.Peter's University, Chennai \\ senthamilkselva@gmail.com \\ ${ }^{2}$ Department of Computer Science Engineering, Chennai \\ lakshmidhevi.b@gmail.com
}

\begin{abstract}
Wireless mesh networking has transpired as a gifted technology for potential broadband wireless access. In a communication network, wireless mesh network plays a vital role in transmission and are structured in a mesh topology. The coordination of mesh routers and mesh clients forms the wireless mesh networks which are routed through the gateways. Wireless mesh networks uses IEEE 802.11 standards and has its wide applications broadband home networking and enterprise networking deployment such as Microsoft wireless mesh and MIT etc. A professional Qos provisioning in intra cluster packet level resource allocation for WMN approach takes power allocation, sub carrier allocation and packet scheduling. This approach combines the merits of a Karush-Kuhn-Tucker (KKT) algorithm and a genetic algorithm (GA) based approach. The KKT algorithm uses uniform power allocation over all the subcarriers, based on the optimal allocation criterion. The genetic algorithm is used to generate useful solutions to optimization and search problems and it is also used for search problems. By combining the intrinsic worth of both the approaches, it facilitates effective QOS provisioning at the packet level. It is concluded that, this approach achieves a preferred stability between system implementation and computational convolution.
\end{abstract}

\section{KEYWORDS}

Wireless Mesh Network (WMN), Karush-Kuhn-Tucker (KKT), Genetic algorithm (GA), Quality of Service $(Q o S)$, Clustering.

\section{INTRODUCTION}

In the networking paradigm, wireless mesh networks brought tremendous changes and is applicable universally. WMNs are structured in a mesh topology. The coordination of mesh routers and mesh clients forms the wireless mesh networks which are routed through the gateways. Our everyday practice of laptop and cell phones act as the mesh clients and mesh routers forwards the signals appropriately through gateways. Wireless mesh networking has been attracting significant attention from industry and academia. WMN affords the internet 
International journal on applications of graph theory in wireless ad hoc networks and sensor networks

(GRAPH-HOC) Vol.5, No.1, March 2013

access with a low cost for all applications. Most of the researchers formulated an absolute work on cross layer information for resource allowance and data scheduling. In [1], adaptive subcarrier, bit and power allocation was investigated to minimize the total transmit power of an OFDM system with fixed data rate. An optimal joint subcarrier and power allocation algorithm was proposed in [5], to maximize the system capacity with fixed power and delay constraints. In [7], resource allocation is performed based on a utility function which reflects the quality of service (QoS). In [6], power allocation of a multimedia CDMA cellular network is determined by maximizing the weighted sum capacity. In [22], an urgency and efficiency based packet scheduling scheme was employed for OFDM systems, based on a utility function of the

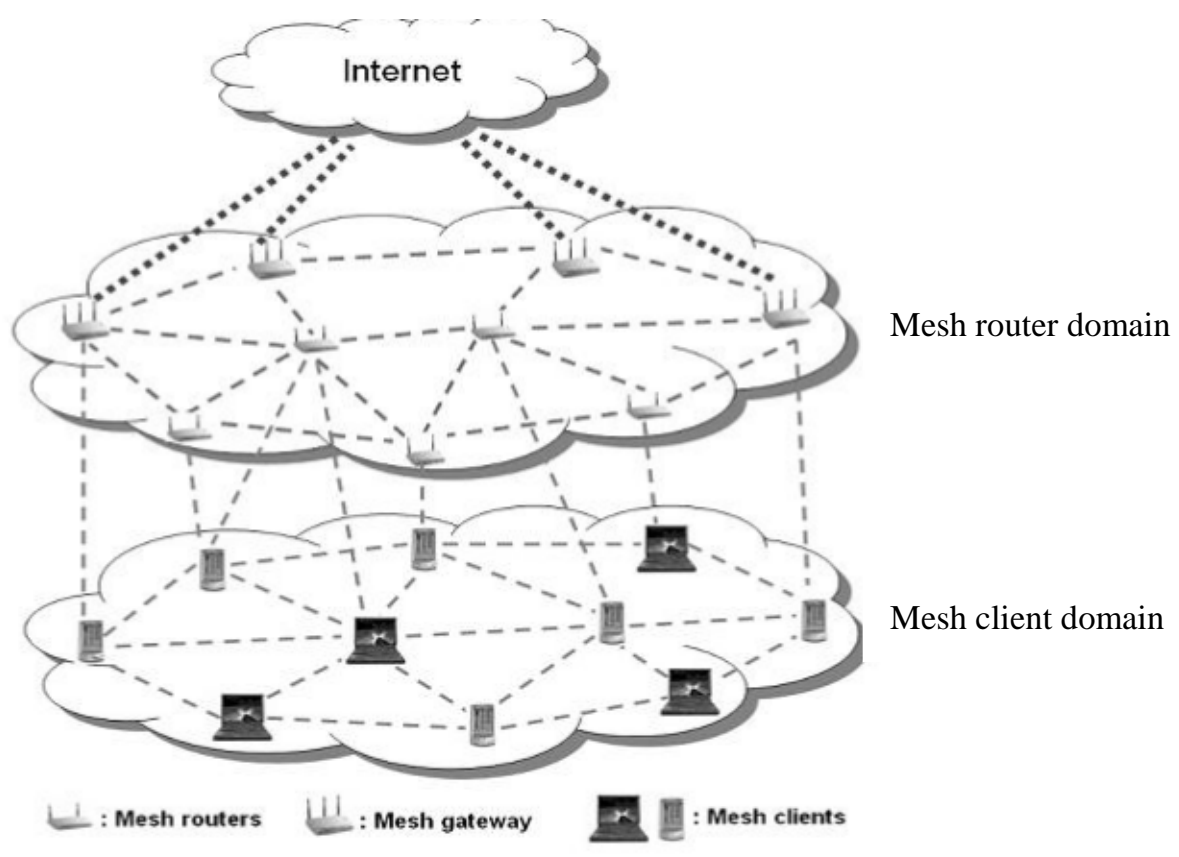

Figure1. Wireless Mesh Network

head-of-line (HOL) packet delay and channel quality. In [24], the genetic algorithm was employed for resource allocation and scheduling by maximizing the weighted sum capacity, where the weights are determined at the MAC layer according to the traffic delays. Our proposed approach concentrates on resource allowance for all the nodes available in clusters. Many technological advances in communication networks offers the services such as conference calling, voice mail and video conferencing etc in MAC layer which necessitates the packet scheduling.

*This paper consists of the following sections. In the section [II] we have report how we designed the related work for an efficient intra clusters packet resources using QOS wireless networks .We report the system model in the section [III], and in the section [IV] The Performance model with the conclusion and the references in the section [V],[VII].* 
International journal on applications of graph theory in wireless ad hoc networks and sensor networks (GRAPH-HOC) Vol.5, No.1, March 2013

\section{RELATED WORK}

In the preceding work, packet scheduling is not addressed properly. The QoS provisioning is often neglected in the MAC layer. To improve the capability of orthogonal frequency division multiplexing systems the cross layer was deliberated in the networks. This work is also different in the following aspects. First, we consider an OFDM system where each user has multiple heterogeneous traffic queues at the same time. Second, we propose a packet dependent (PD) scheduling scheme, which determines the packet transmission order by assigning different weights to different packets, and therefore is more efficient than the conventional queue dependent scheduling [20][8] where all packets in a queue have the same weight. The packet weights in PD scheduling are determined based on the delay, packet size and QoS priority level of the packets. Third, our resource allocation is user based, by maximizing the weighted sum capacity of users rather than queues, and therefore requires a lower complexity than the conventional queue based resource allocation. The weight for each user is obtained by summing the weights of selected packets for that user.

Regarding the problem of power-subcarrier-time resource allocation with QoS assurance, a KKTdriven resource allocation algorithm is proposed in [10], effectively provisioning QoS at the packet level (e.g., packet dropping rates).This simple KKT-driven algorithm is shown to outperform a random scheme. KKT-driven approach considers all three resource dimensions (i.e., power, subcarrier, and time) individually in succession. Thus, the throughput performance can be quite far from optimal. Genetic algorithms (GAs) are commonly used as search algorithms for near-optimal solutions [12]. GA-based resource allocation can incorporate all three resource dimensions simultaneously, potentially achieving better throughput performance and provisioning QoS more effectively. Yet, GAs are usually computationally expensive due to a long convergence time.

\section{SYSTEM MODEL}

In this work, WMN is considered and it is a package of more clusters. Each and every node in the communication networks are connected together. Grouping of similar nodes in to clusters are called clustering and this technique is employed. By similarity the nodes can be split up into small groups which are referred to as clusters for effective data combination. For all the clusters available in the networks, must have a cluster head

for directing the other nodes to perform the data communication effectively. This cluster head must have adequate resources and it was elected by the neighboring nodes. By employing the clustering mechanism, the networks can provide bandwidth, stability and scalability. The nodes in the cluster may vary or it may be constant according to the traffic load increase or decrease. The cluster head plays an important role and this offers the resource allowance for all the lively nodes in the cluster. Also it affords the timing information to all the clusters available in the network. Further this time is divided into frames. Every frame in the nodes consists of a beacon slot, control slot and data slots in a serial manner which is shown in figure 2. 
International journal on applications of graph theory in wireless ad hoc networks and sensor networks

(GRAPH-HOC) Vol.5, No.1, March 2013

Figure 2. Frame structure

\begin{tabular}{|l|l|l|l|l|l|l|}
\hline Beacon slot & Control slot & Data 1 & Data 2 & Data 3 & $\ldots . . .$. & Data n \\
\hline
\end{tabular}

Each and every slot in the frame can have their own functionality. The beacon slot in the frame provides timing and node cluster sequence which are needed for transmission in the data slots. The control slot in the frame

reveals the needed information of resource allowance technique to the beacon slot for all the clusters in the network. A mesh router can be a sender, relay or receiver at different times. The mesh routers act as an Omni-directional transceiver.

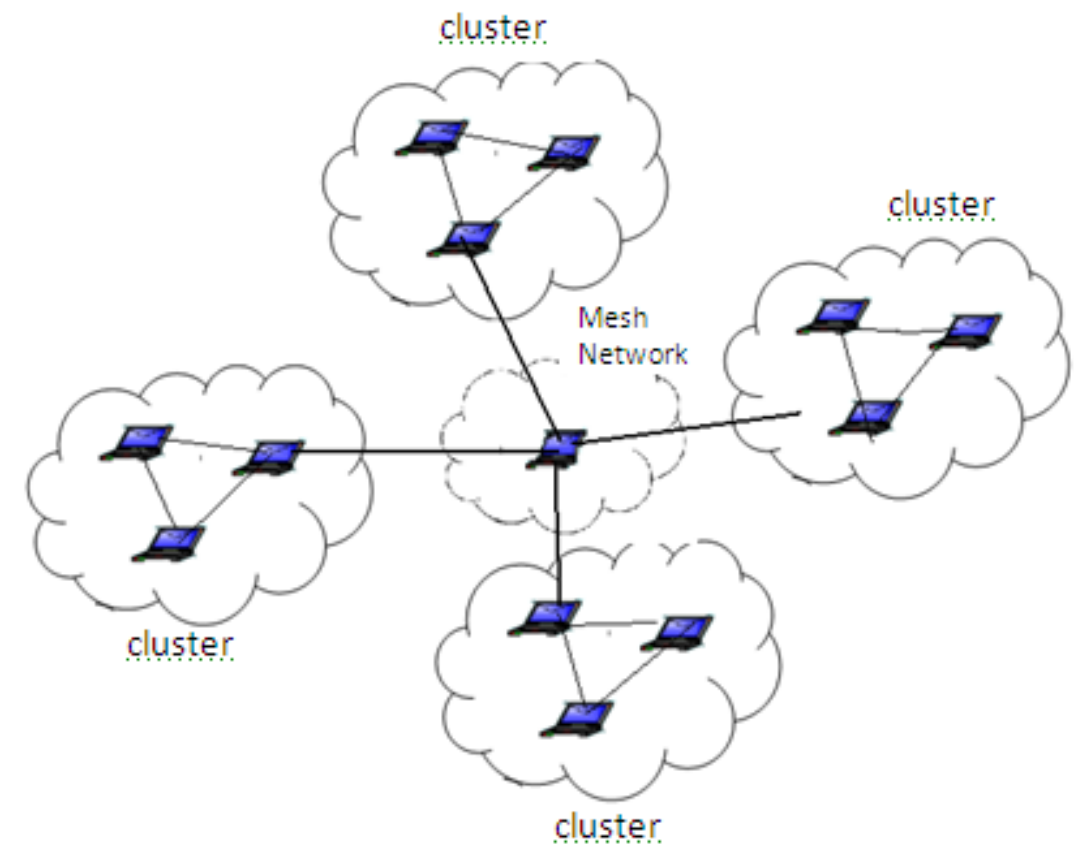

Figure 3. An Intra-cluster WMN

Orthogonal frequency division multiplexing (OFDM), which divides a broadband channel into a set of orthogonal narrowband subcarriers, allows for a wide range of resource management approaches, and therefore is regarded as a promising technology for future wireless communications. With the help of multi-channel OFDM technology, each router can choose a set of subcarriers for DATA transmissions and/or receptions. In order to focus on intra-cluster resource allocation, we make the following assumptions for presentation clarity. The first focus is on Qos satisfaction in the networking attributes such as cell loss ratio, cell transfer delay, cell delay variation and cell error ratio. The second focus is on clustering mechanism for effective packet scheduling. 
International journal on applications of graph theory in wireless ad hoc networks and sensor networks

(GRAPH-HOC) Vol.5, No.1, March 2013

\section{PROBLEM FORMULATION}

In our problem formulation, we take the instantaneous rate requirements of different traffic types (e.g., voice, video, and data) in the current frame, if any, into account. Let $X, Y$, and $Z$ denote the number of active links in a cluster, the number of subcarriers available in a cluster, and the number of timeslots (i.e., DATA slots) in a frame, respectively. Consider the following resource allocation optimization problem which is the selection of the best element and is given below. In an optimization problem solving, we must calculate the maximizing function and minimizing function by systematical computation approach function.

$$
\begin{gathered}
\max \sum_{y=1}^{\mathrm{X}}\left(U_{X}\left(R_{X}(\mathrm{a}, \mathrm{p}) .\right)\right. \\
\text { subject to } R_{X}(\mathrm{a}, \mathrm{p})>=\mathrm{R}_{\mathrm{m}}^{\mathrm{d}}, \forall \mathrm{m} \\
\max \sum_{y=1}^{\mathrm{Y}}\left(P_{x, y=} P_{x, \max }\right), \forall \mathrm{m}, 1
\end{gathered}
$$

Here, Um is the utility function of the $X$ link. $R x(\mathrm{a}, \mathrm{p})$ represents the actual aggregate transmission rate of the frame allocating the $\mathrm{Y}$ subcarriers to all the active links in the cluster. The transmit power over all the sub carriers in the transmitter link should be indicated by $\mathrm{p}$. The maximum power constraint of the transmitter and the channel gain should be indicated for all the links. By using the resources allocated, the particular content of data should be transmitted without any loss and noise interruption.

\section{COMBINED KKT-GA RESOURCE ALLOCATION}

Karush-Kuhn-Tuker algorithm first uses uniform power allocation over all the subcarriers, allocates subcarriers based on the optimal subcarrier allocation criterion, re-allocates the subcarriers until all the system constraints are met, and finally performs water- filling for power re-allocation. In a multi-carrier communication system, each channel can carry a signal under noise condition considering maximizing the transmission rate and minimizing the total power consumption. The KKT algorithm finds the solution for optimization problems constrained to one or more inequalities. This algorithm follows some necessary conditions while finding the solution: (i) Feasibility (ii) Direction which improves objective (iii) Complementary slackness (iv) Positive Lagrange multipliers. The time complexity of the Cheng's KKT-driven algorithm is on the order of $\mathrm{O}(\mathrm{LMN})$. Despite low complexity, this approach is only suboptimal, because it considers three resource dimensions (i.e., power, subcarrier, and time) individually in succession .In our project this algorithm is used for allocation process for the optimal allocation of a node to the server (cluster header) based on the above three resource dimensions power, subcarrier, time.

The genetic algorithm (GA) is a search heuristic that mimics the process of natural evolution. This heuristic is routinely used to generate useful solutions to optimization and search problems. In our project genetic algorithm is used for selection process and fitness function evaluation. A typical genetic algorithm describes the genetic representation of the solution and it represents the 
International journal on applications of graph theory in wireless ad hoc networks and sensor networks

(GRAPH-HOC) Vol.5, No.1, March 2013

string of bits which describes the characteristics of the genetic illustration. A fitness function evaluates the strings of the solution by fixing the fitness functionality value. By employing reproducing and breeding, we can obtain the best elucidation. So that avoiding the unwanted or useless solution in further.

Genetic algorithms are a type of trial-and-error search technique that is guided by principles of Darwinian evolution. The genetic algorithm follows the concept of living organisms in natural evolution, but considering the string values following selection, mutation and reproduction. GAs start with a pool of randomly-generated candidate solutions which are then tested and scored with respect to their utility. Solutions are then bred by probabilistically selecting high quality parents and recombining their genetic representations to produce offspring solutions. Offspring are typically subjected to a small amount of random mutation. The process of following selection, mutation and recombination must be enforced continuously till obtaining the acceptable solution. Genetic algorithms has its wide applications in variety of areas such as micro-biology, medicine and in almost all authorities.

Parallelized versions of genetic algorithms (GAs) are popular primarily for three reasons: the GA is an inherently parallel algorithm, typical GA applications are very compute intensive, and powerful computing platforms, especially Beowulf-style computing clusters, are becoming more affordable and easier to implement. In addition, the low communication bandwidth required allows the use of inexpensive networking hardware such as standard office Ethernet.

In our work, we have the center of attention to perform the data communication without any packet data loss and sustains the system implementation in the best manner. The time complexity of Combined-KKT-GA is low, resulting in a preferred candidate for practical implementation. We propose to combine the Cheng's KKT driven and GA-based resource allocation schemes as follows.

1) Obtain a resource allocation solution using the Cheng's KKT-driven approach.

2) Obtain a resource allocation solution using the GA based approach.

3) Choose the better solution out of the above two solutions.

Figure 4. KKT-GA algorithm combined flowchart

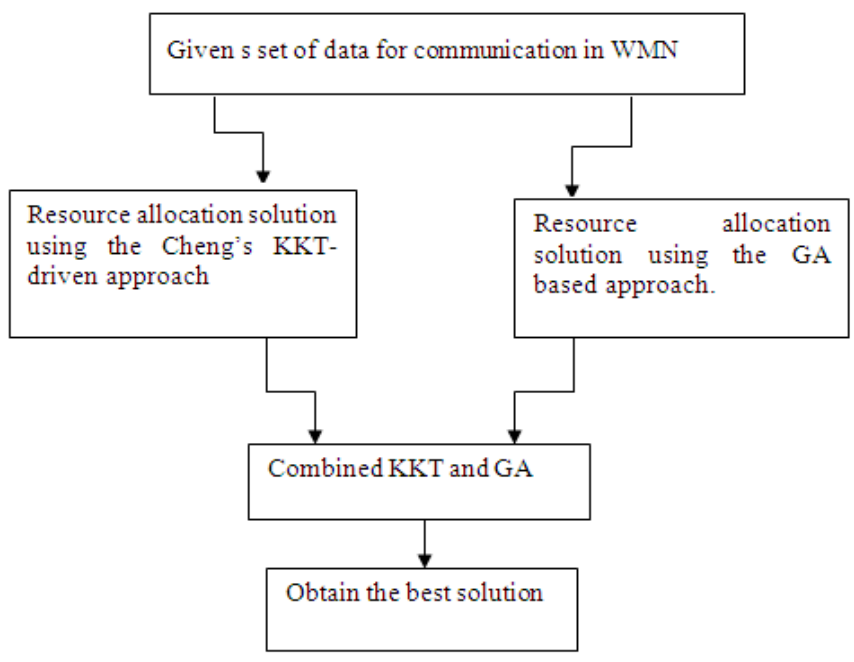


International journal on applications of graph theory in wireless ad hoc networks and sensor networks

(GRAPH-HOC) Vol.5, No.1, March 2013

Therefore, on average, the performances of this novel approach. Combined-KKT- GA is expected to be lower bounded and upper bounded by that of the Cheng's KKT-driven scheme and that of the GA-based scheme, respectively.

\section{PERFORMANCE EVALUATION}

We consider a cluster with a number of wireless nodes randomly located in a $1 \mathrm{~km} \times 1 \mathrm{~km}$ coverage area. We assume that the routing is predetermined so that the transmission source and destination pair of an incoming packet is known in advance In Figure. 6, there is an obvious performance gap between the system throughputs obtained from all the approaches and the data capacity. This gap is recognized to the low traffic load and resource reservation. We observe that both the system throughput and resource utilization for the proposed algorithms are improved when the traffic load increases. The proposed Combined-KKTGA approach can effectively provision QoS for real-time traffic and achieve satisfactory system throughput performance. This novel approach is also of low computational complexity, leading to a viable candidate for practical implementation

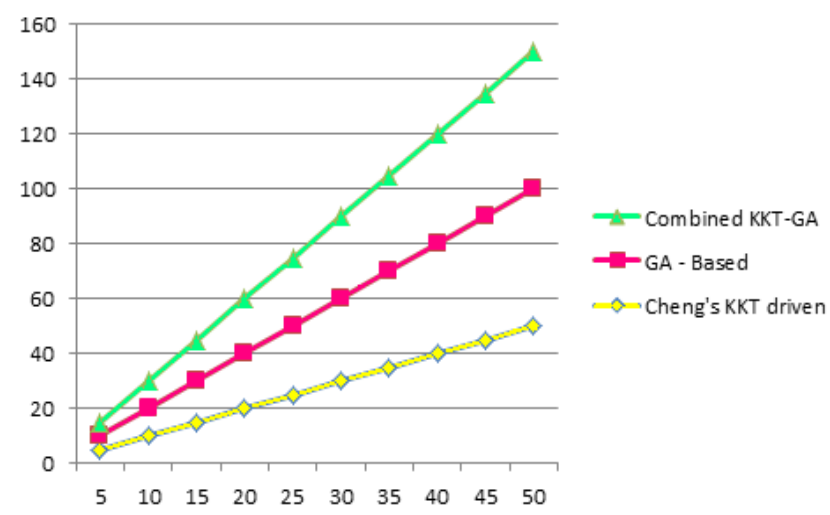

Figure 5. System throughputs of the proposed scheme (Combined-KKT-GA), the GA-based scheme, the Cheng's KKT-driven scheme for the number of links M.

\section{RESULTS}

In a wireless communication mesh network, devoted links are used that gives the assurance of holding their own information and avoids the traffic tribulations and this mesh network is robust on the whole networks. This WMN provides the system performance, accuracy of data without any packet data loss and deliver data in a timely manner. When the data communication occurs between the client nodes, the server must be initiated in prior and intimates the client to sends the data which is shown in figure 7.Now the client is ready to transfer the files, on receiving the server ip address which is shown in figure 8 . So the file transmission can be done in the client window by mentioning the appropriate file name which is shown in figure 9. The data must be received by the destination with-in a second and the file contents are transferred accurately without any significant delay. 
International journal on applications of graph theory in wireless ad hoc networks and sensor networks (GRAPH-HOC) Vol.5, No.1, March 2013

Figure6. The sever started message box

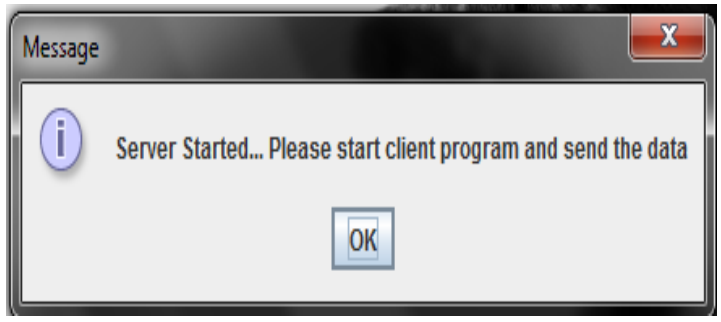

Figure 7. Output response given by the server message box

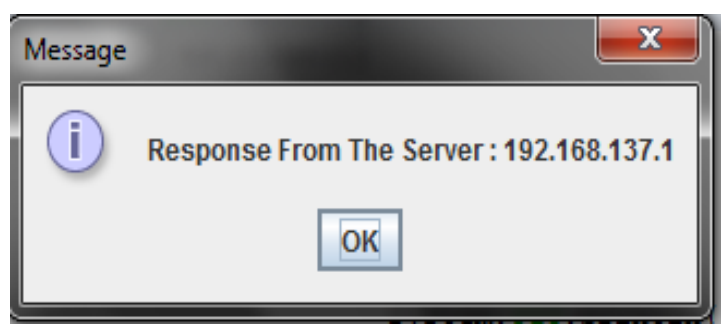

Figure 8. Client side window

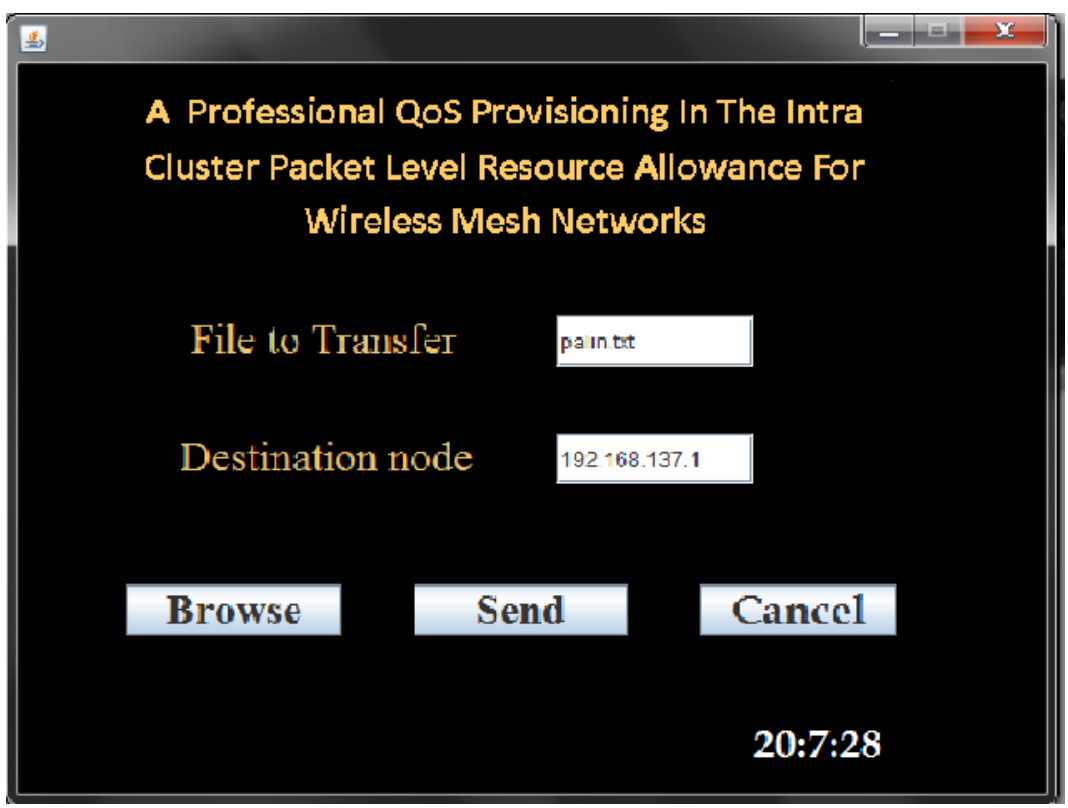


International journal on applications of graph theory in wireless ad hoc networks and sensor networks

(GRAPH-HOC) Vol.5, No.1, March 2013

Figure 9. After specifying file name, the client transfers content to the server

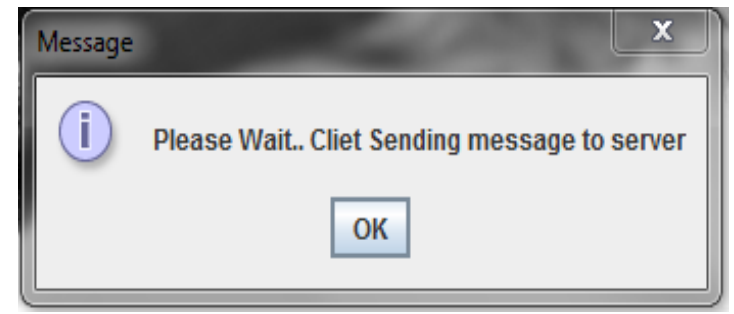

Figure 10. The Destination side window

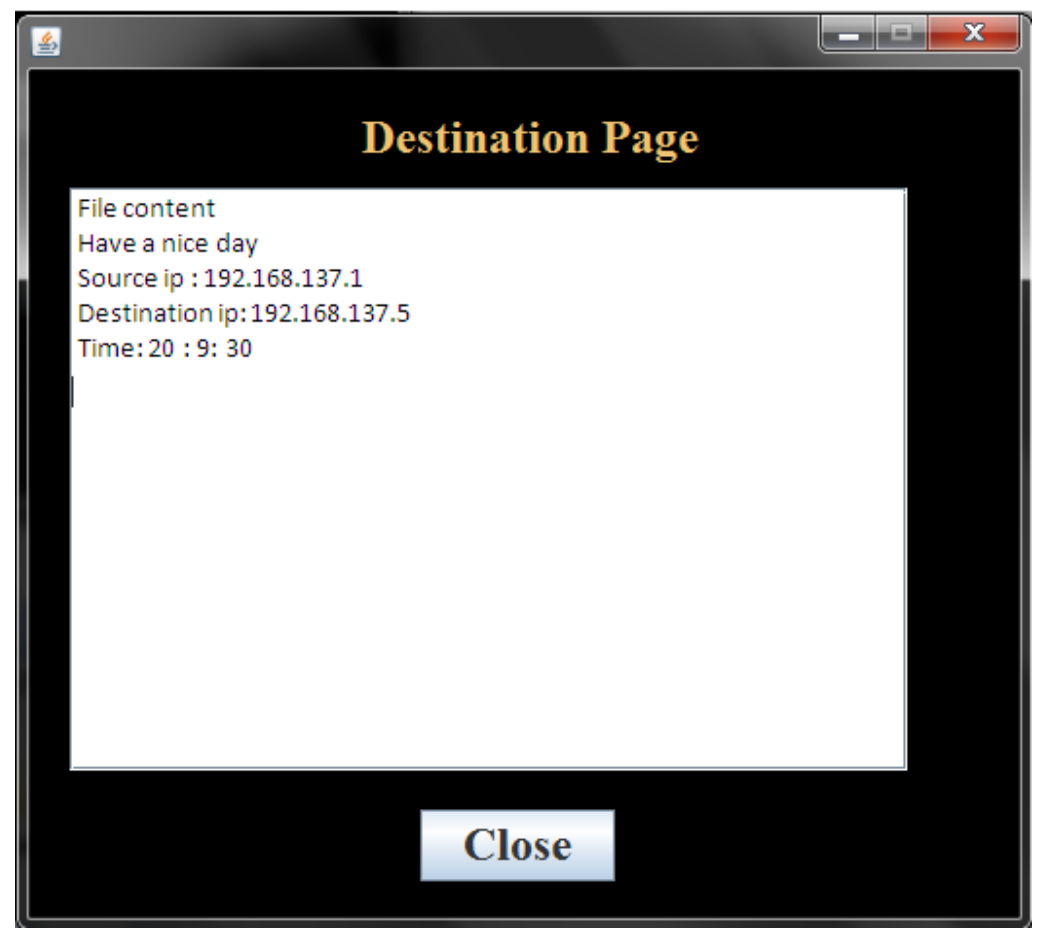

\section{CONCLUSION}

In this work, by combining the intrinsic worth of KKT algorithm and genetic algorithm that facilitates professional QOS provisioning in the intra cluster packet level. This combined algorithm is proposed for the joint power subcarrier- time intra-cluster resource allocation problem in WMNs. This proposed approach provides reliability, privacy, security that makes fault identification and fault isolation easily. It is concluded that, this approach achieves a preferred stability between system implementation and computational convolution. 
International journal on applications of graph theory in wireless ad hoc networks and sensor networks

(GRAPH-HOC) Vol.5, No.1, March 2013

\section{REFERENCES}

[1] C. Y. Wong, R. S. Cheng, K. B. Lataief, and R. D. Murch, "Multiuser OFDM with adaptive subcarrier, bit, and power allocation," IEEE J.Select. Areas Commun. vol. 17, no. 10, pp. 1747-1758, Oct. 1999.

[2] D. S. W. Hui, V. K. N. Lau, and W. H. Lam, "Cross-layer design for OFDMA wireless systems with heterogeneous delay requirements,'IEEE Trans. Wireless Commun., vol. 6, pp. 2872-2880, Aug. 2007.

[3] G. C. Song, "Cross-layer resource allocation and scheduling in wireless multicarrier networks," Ph.D. dissertation, Georgia Institute of Technology, 2005.

[4] H. T. Cheng, H. Jiang, and W. Zhuang, "Distributed medium access control for wireless mesh networks," Wireless Commun. Mobile Computing, vol. 6, no. 6, pp. 845-864, Sept. 2006.

[5] H. T. Cheng and W. Zhuang, "Pareto optimal resource management for wireless mesh networks with QoS assurance: joint node clustering and subcarrier allocation," IEEE Trans. Wireless commun., in press.

[6] H. Yin and H. Liu, "An efficient multiuser loading algorithm for OFDM based broadband wireless systems," in Proc.IEEE Globecom'00, vol. 1, 2000, pp. 103-107.

[7] L. Gao and S. Cui, "Efficient subcarrier, power, and rate allocation with fairness consideration for OFDMA uplink,"IEEE Trans. WirelessCommun., vol. 7, no. 5, pp. 1507-1511, May 2008.

[8] M. Andrews, K. Kumar an, K. Ramanan, A. Stolyar, P. Whiting, and. Vijayakumar, "Providing quality of service Over a shared wireless link," IEEE Commun. Mag., vol. 2, pp. 150-154, Feb. 2001.

[9] M. Mitchell, An Introduction to Genetic Algorithms. Cambridge, MA:The MIT Press, 1998.

[10] Y. Yu and W. Zhou, "Resource allocation for OFDMA system based on genetic algorithm," in Proc. IEEE IWCLD’2007, vol. 10, Tai’an, China,Sep. 2007, pp. 65-69.

\section{BIOGRAPHIES}

K.Senthamil Selvan has completed his B.E. in the faculty of ECE and M.E. in the area of Applied Electronics. He is currently a research scholar in the department of Electronics and Communication Engineering at St. Peter's University, Avadi, Chennai. He is an Assistant Professor in the dept. of ECE at Dhanalakshmi College of Engineering, Chennai. He shows interest in the areas of Wireless Communication, Embedded system design and Image processing.

B.Lakshmi Dhevi has completed her B.E. in the faculty of Computer Science and Engineering (CSE), M.E. in the area of Computer Science and Engineering. She shows interest in the areas of Wireless sensor networks, Cloud Computing and Image processing.
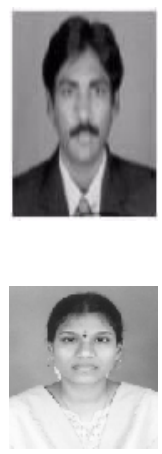